\title{
SPITZER OBSERVATIONS OF DUST DESTRUCTION IN THE PUPPIS A SUPERNOVA REMNANT
}

\author{
Richard G. Arendt ${ }^{1,2}$, Eli Dwek ${ }^{2}$, William P. Blair ${ }^{3}$, Parviz Ghavamian ${ }^{4}$, Una Hwang ${ }^{3,5}$, \\ Knox S. Long ${ }^{4}$, Robert Petre ${ }^{5}$, Jeonghee Rho ${ }^{6,7}$, and P. Frank Winkler ${ }^{8}$ \\ ${ }^{1}$ CRESST, University of Maryland-Baltimore County, Baltimore, MD 21250, USA; Richard.G.Arendt@ nasa.gov \\ 2 NASA Goddard Space Flight Center, Code 665, Greenbelt, MD 20771, USA \\ ${ }^{3}$ The Henry A. Rowland Department of Physics and Astronomy, Johns Hopkins University, 3400 North Charles Street, Baltimore, MD 21218, USA \\ ${ }^{4}$ Space Telescope Science Institute, 3700 San Martin Drive, Baltimore, MD 21218, USA \\ ${ }^{5}$ NASA Goddard Space Flight Center, Code 662, Greenbelt, MD 20771, USA \\ ${ }^{6}$ Spitzer Science Center, MS 220-6, California Institute of Technology, Pasadena, CA 91125, USA \\ ${ }^{7}$ Universities Space Research Association, NASA Ames Research Center, MS 211-3, Moffett Field, CA 94035, USA \\ ${ }^{8}$ Department of Physics, Middlebury College, Middlebury, VT 05753, USA \\ Received 2010 August 5; accepted 2010 September 30; published 2010 November 19
}

\begin{abstract}
The interaction of the Puppis A supernova remnant (SNR) with a neighboring molecular cloud provides a unique opportunity to measure the amount of grain destruction in an SNR shock. Spitzer Space Telescope MIPS imaging of the entire SNR at 24, 70, and $160 \mu \mathrm{m}$ shows an extremely good correlation with X-ray emission, indicating that the SNR's IR radiation is dominated by the thermal emission of swept-up interstellar dust, collisionally heated by the hot shocked gas. Spitzer IRS spectral observations targeted both the Bright Eastern Knot (BEK) of the SNR where a small cloud has been engulfed by the supernova blast wave and outlying portions of the associated molecular cloud that are yet to be hit by the shock front. Modeling the spectra from both regions reveals the composition and the grain size distribution of the interstellar dust, both in front of and behind the SNR shock front. The comparison shows that the ubiquitous polycyclic aromatic hydrocarbons of the interstellar medium are destroyed within the BEK, along with nearly $25 \%$ of the mass of graphite and silicate dust grains.
\end{abstract}

Key words: dust, extinction - infrared: ISM - ISM: individual objects (Puppis A) - ISM: supernova remnants

Online-only material: color figures

\section{INTRODUCTION}

The supernova remnant (SNR) shock processing of interstellar dust plays an extremely important role in determining dust composition, abundance, and size distribution, which in turn have a great effect on redistributing starlight, regulating the temperature of most of the diffuse gas in the interstellar medium (ISM), and determining the rates and efficiencies of chemical reactions in molecular clouds. Compensating for these destruction processes in the diffuse ISM are various gas-grain and grain-grain interactions that grow icy and refractory organic mantles on the surviving dust grains in the denser phases of the ISM.

Determining the efficiency of the various grain destruction processes is of great astrophysical importance since they play a major role in our understanding of the sources and origin of interstellar dust. Knowledge of the grain destruction efficiency is crucial for determining the origin of the large amount of dust observed in the early universe (Dwek et al. 2007), whether it could all be formed by supernovae (SNe; Morgan \& Edmunds 2003; Maiolino et al. 2004), or in AGB stars (Valiante et al. 2009; Cherchneff \& Dwek 2010). It also plays an important role in understanding the dust abundance and the origin of the elemental depletion pattern in the local universe (Jones et al. 1996; Jones 2004; Tielens 1998; Dwek 1998).

Only in the youngest SNRs (ages $\lesssim 1000$ yr) is the emission still dominated by the ejecta of the SN. Emission of most SNRs is instead dominated by the swept-up ISM. The ideal measurement of dust processing in shocks is to examine a population of ISM dust before and after passage through an SNR shock front. While many SNRs exhibit post-shock IR emission (Arendt 1989; Saken et al. 1992; Reach et al. 2006), only a fraction of those have clear indication of interaction with specifically identifiable clouds in the surrounding ISM. Of these, the Puppis A SNR is probably the clearest case in which the cloud being encountered exhibits identifiable IR emission.

Puppis A is a relatively young SNR with an age of $\sim 3700 \mathrm{yr}$ (Winkler et al. 1988) and lying at a distance of $\sim 2.2 \mathrm{kpc}$ (Dubner \& Arnal 1988; Reynoso et al. 1995). It is a strong X-ray source dominated by the shocked ISM, but there are isolated features in the SNR that can be identified as SN ejecta (Hwang et al. 2008; Katsuda et al. 2008, 2010). The synchrotron radio emission is similar in structure to the X-ray emission, showing a partial shell significantly brighter on the northeast side, but the two are not well correlated in detail (Castelletti et al. 2006). Optical emission has an entirely different morphology. The brightest features are a ridge of filaments along the shock front on the NW side of the SNR (Baade \& Minkowski 1954; Goudis \& Meaburn 1978). On a large scale, these coincide with the radio and $\mathrm{X}$-ray emission, though small-scale details differ. These filaments have very strong [ $\left.\mathrm{N}_{\text {II }}\right]$ emission, indicating that circumstellar material is being shocked. Optical emission elsewhere in the SNR consists of more randomly scattered knots and filaments. Some of the optical knots exhibit O-rich spectra that characterize them as SN ejecta (Winkler \& Kirshner 1985; Sutherland \& Dopita 1995). The kinematics of these ejecta knots provide age and distance estimates of the SNR. Distance estimates are also provided by the Galactic rotation distances of observed $\mathrm{HI}$ and CO clouds that are associated with the SNR (Dubner \& Arnal 1988; Reynoso et al. 1995; Paron et al. 2008).

At IR wavelengths Puppis A was first observed as part of the IRAS full-sky survey (Neugebauer et al. 1984). Puppis A was bright and well resolved from the confusion of the background ISM at 25 and $60 \mu \mathrm{m}$, while emission from the SNR was much 
more difficult to distinguish at 12 and $100 \mu \mathrm{m}$. At arcminute resolution, the IR emission was observed to be well correlated with the X-ray emission (Arendt et al. 1990). This is perhaps the best such correlation of any Galactic SNR. The brightest feature at IR (and X-ray) wavelengths is a compact feature known as the Bright Eastern Knot (BEK). The BEK is at an indentation in the perimeter of the SNR where the SNR is in contact with a neighboring molecular cloud (Dubner \& Arnal 1988), known as the "East Cloud." The East Cloud is distinguishable in the broadband IR imaging, which is a rarity for clouds adjacent to SNRs. In most other cases of SNR-cloud interaction, the cloud is confused with all the other clouds along the same line of sight. Using the IRAS data, Arendt et al. (1991) had compared the broadband spectral energy distribution (SED) of the BEK with that of the East Cloud to estimate that as much $\sim 30 \%$ of the mass of swept-up dust was being destroyed. However, with only the limited broadband SED provided by the IRAS data, there were no direct confirmations of the composition of the pre- or post-shock dust, or that the IR emission was unaffected by any significant line emission components.

Thus, observations of Puppis A were undertaken with the Spitzer Space Telescope (Werner et al. 2004; Gehrz et al. 2007) to provide a complete high spatial resolution map of the SNR and the clouds with which it is interacting at 24,70 , and $160 \mu \mathrm{m}$, and to obtain 5-40 $\mu \mathrm{m}$ spectra of the interstellar dust before and after it is swept up by the SNR. Section 2 of this paper describes these observations and the data reduction. The resulting images and spectra are presented in Section 3, along with models which can be used to characterize the changes in the dust grain size distribution induced by the passage though the shock and into a hot dense environment. In Section 4, we discuss the implied amount of grain destruction and the relations between the gas temperature and density (or ionization timescale) as derived independently from the IR and X-ray modeling. We summarize our results in Section 5.

\section{SPITZER OBSERVATIONS AND DATA REDUCTION}

The Spitzer observations were carried out under program PID 50270. The observations employed all three instruments on Spitzer: the Multiband Imaging Photometer for Spitzer (MIPS; Rieke et al. 2004), the InfraRed Array Camera (IRAC; Fazio et al. 2004), and the InfraRed Spectrograph (IRS; Houck et al. 2004).

\subsection{MIPS}

The entire SNR and the East Cloud were observed using MIPS. Two Astronomical Observation Requests (AORs: $27514624,27515136)$ with fast $1^{\circ}$ scan legs and $148^{\prime \prime}$ offsets provided complete coverage at 24 and $70 \mu \mathrm{m}$. The total integration time per pixel on the sky was $31.4 \mathrm{~s}$ and $15.7 \mathrm{~s}$ at 24 and $70 \mu \mathrm{m}$, respectively. Due to the fast scan rate and bad pixels in the $160 \mu \mathrm{m}$ detector array, there were periodic gaps in the $160 \mu \mathrm{m}$ coverage.

The individual frames generated from each AOR were processed by the Spitzer Science Center (SSC) to produce the Basic Calibrated Data (BCD). The SSC also ran a post-BCD pipeline that mosaicked the individual frames of the $\mathrm{BCD}$ into a single mosaic for each AOR. The post-BCD mosaics for the $24 \mu \mathrm{m}$ data were very good. Therefore our processing of these data merely consisted of reprojecting the two AORs to a common grid (2".45 pixels in the scan orientation) and constructing a weighted average of the AORs using the given weights. Addi- tional variants of this image were then created with pixel scales and resolution to match the 70 and $160 \mu \mathrm{m}$ data, and in celestial coordinates.

The post-BCD mosaics for the $70 \mu \mathrm{m}$ data were not suitable for use. The $70 \mu \mathrm{m}$ BCD frames data were reprocessed using the least-squares self-calibration procedure of Fixsen et al. (2000). As applied here, the procedure represents the data at each pixel $i$ of each frame, $D^{i}$, as $D^{i}=S^{\alpha}+F^{p}+F^{q}$, where $S^{\alpha}$ is the intensity of the sky at location $\alpha, F^{p}$ is a detector offset for detector pixel $p$ (constant for all frames), and $F^{q}$ is another offset which is constant for all detector pixels but varies from frame to frame $q$. The self-calibration procedure is then applied to derive the detector calibration parameters, $F^{p}$ and $F^{q}$. As we began with the individual BCD frames, these detector offsets represent delta-corrections that account for transient or temporary effects that were not properly removed by the standard BCD pipeline. These offsets were subtracted from the data $D^{i}$, and then these corrected BCD frames were remapped to obtain the self-calibrated sky map $S^{\alpha}$. This procedure worked well to remove residual responses after the periodic stim flashes and to tie together the different scan legs in a consistent manner. However, there was still a periodic striping apparent in the images. This striping could be mitigated by median filtering the data in the time domain, before the self-calibration is performed. The effect of the width of the median filter was investigated. Narrower filters produced cleaner results but at the expense of removing some of the large-scale emission; wider filters were not as effective in removing the striping. Intermediate values were not a satisfactory balance. Thus, the $70 \mu \mathrm{m}$ mosaic presented here was created by Fourier transforming a narrowfiltered mosaic and a wide-filtered mosaic and combining the small-scale structure $(<4.7)$ from the former with the largescale structure $(>4.7)$ of the latter, before performing the inverse Fourier transform. The result is far cleaner than the post-BCD mosaics. However, there are some linear structures in the SNR that happen to be aligned with the scan direction and appear to be filtered out by this procedure. Some of these are real, as they have clear $24 \mu \mathrm{m}$ counterparts, but in other cases the features are artifacts that are better removed. Thus, some care is needed in the quantitative analysis of the $70 \mu \mathrm{m}$ emission.

Significant gaps and striping in the $160 \mu \mathrm{m}$ data presented an even greater challenge. The standard post-BCD mosaics provided by the SSC were strongly striped. The standard filtered versions, which are also provided by the SSC, were free of striping, but also clearly lacked large-scale structure in the emission as compared to the unfiltered versions. Both mosaics contained gaps. Thus, the first step in our processing was to interpolate across the gaps in the filtered post-BCD mosaics by using a distance-weighted average of all good pixels within a 5 pixel radius of each missing pixel (weight $=1 /$ distance $^{2}$ ). To restore the large-scale structure, a fifth-order polynomial background was fit to the unfiltered post-BCD mosaic and then added to the "filled" version of the filtered post-BCD mosaic. This was performed on the two AORs independently. The AORs were reprojected to a common grid and combined using a weighted average, as was done for the $24 \mu \mathrm{m}$ data. The general result appears good, with the caveat that the large-scale structure may be inaccurate.

\subsection{IRAC}

IRAC observations were much more limited than the MIPS observations, and only targeted a single $5^{\prime} \times 5^{\prime}$ field of view at the location of the BEK where the spectroscopy was to 
be obtained. Two pointings were required to obtain coverage at all four IRAC wavelengths, so adjacent $5^{\prime} \times 5^{\prime}$ files were serendipitously imaged to the north of the BEK at 4.5 and $8 \mu \mathrm{m}$ and to the south at 3.6 and $5.8 \mu \mathrm{m}$. Each set of pointings used $12 \mathrm{~s}$ frame times and a large 12-point Reuleaux dither pattern to reach a nominal integration time of $124.8 \mathrm{~s} \mathrm{pixel}^{-1}$ and nominal sensitivities of $0.02,0.02,0.07$, and $0.09 \mathrm{MJy} \mathrm{sr}^{-1}$ at 3.6, 4.5, 5.8 , and $8 \mu \mathrm{m}$, respectively. Being the brightest portion of the SNR at $24 \mu \mathrm{m}$, this would be the most likely location to detect any thermal emission at 3.6-8 $\mu \mathrm{m}$. Also the BEK is one of the relatively few places in the SNR where there are optical lineemitting filaments, which may have IR counterparts at these wavelengths. The data from the single AOR (27514112) were processed using the least-squares self-calibration (Fixsen et al. 2000).

\subsection{IRS}

The primary IRS data were collected as two spectral maps using both orders of the long low module (LL1 and LL2; AOR 27515136) and both orders of the short low module (SL1 and SL2; AOR 27514368). The area covered jointly by both modules in all four spectral orders is $\sim 3.5 \times 3.5$, with additional outlying coverage in different directions for different orders. The LL spectral map completely covered the region. However, we chose to undersample the SL spectral map in the cross scan direction to avoid the excessive amount of observing time that would be required to fully cover the desired field. A third AOR (27514880) used staring mode to target a cluster of seven positions (three locations in the East Cloud and four background fields) with the same integration times that were used in the spectral maps. The data from each AOR were reduced and spectra for selected regions were extracted using CUBISM (Smith et al. 2007). However, the reported uncertainties were clearly smaller than the dispersion in the spectra as a function of wavelength. Therefore, the CUBISM uncertainties were renormalized (by factors of $\sim 3$ ) such that they matched the empirical dispersion of the spectra. CUBISM was also used to produce spectral line maps for emission lines of [Ne II] $12.8 \mu \mathrm{m}$, [Fe II] $17.9 \mu \mathrm{m},[\mathrm{Fe}$ II]+[O IV] $26 \mu \mathrm{m},[\mathrm{Ne}$ III] $15.6 \mu \mathrm{m}$, [S III] $18.7 \mu \mathrm{m}$, and [S III] $33.5 \mu \mathrm{m}$. For the LL spectra, the background was selected from the nearest of the background pointings outside the SNR at $(\alpha, \delta)=\left(8^{\mathrm{h}} 24^{\mathrm{m}} 53^{\mathrm{s}} .7,-42^{\circ} 58^{\prime} 16^{\prime \prime}\right)$ (J2000). At the SL wavelengths, this location was clearly too bright to use as a background at the location of the BEK. Thus for the SL spectra, the average of two more distant, but darker, locations was used $(\alpha, \delta)=\left(8^{\mathrm{h}} 25^{\mathrm{m}} 32^{\mathrm{s}} .9,-43^{\circ} 07^{\prime} 32^{\prime \prime}\right)$, $\left(8^{\mathrm{h}} 25^{\mathrm{m}} 23^{\mathrm{s}} .7,-42^{\circ} 48^{\prime} 24^{\prime \prime}\right)$.

No extinction corrections were applied to the data. The integrated $\mathrm{H}$ I column density toward the BEK in the velocity range $0-16 \mathrm{~km} \mathrm{~s}^{-1}$ is $\sim 0.15 \times 10^{22} \mathrm{~cm}^{-2}$ (Dubner \& Arnal 1988), while $X$-ray modeling of the BEK indicates column densities from this value up to $\sim 0.4 \times 10^{22} \mathrm{~cm}^{-2}$. These are fairly typical values for a $2.2 \mathrm{kpc}$ line of sight in the Galactic plane. Using the extinction curve of Mathis (1990) to convert the column densities, we find that the most extreme extinction is $A_{\lambda}<0.13$ at the $9.7 \mu \mathrm{m}$ silicate absorption feature, with $A_{\lambda}$ being several times lower at other wavelengths from 5 to $40 \mu \mathrm{m}$.

\section{RESULTS}

\subsection{Images}

A color image of the Puppis A SNR at 24 and $70 \mu \mathrm{m}$ and $\mathrm{X}$-rays is shown in Figure 1. The separate MIPS images of the
SNR and surrounding ISM are shown in Figure 2. Puppis A is a large, bright, and highly structured source at 25 and $70 \mu \mathrm{m}$, with an extent of $\sim 50^{\prime}$. There is a general gradient in brightness, decreasing from the northeast to the southwest, which coincides with increasing distance from the Galactic plane. The bulk of the emission consists of filamentary structures that often appear to be composed of shorter $\left(\sim 1^{\prime}\right)$ arced segments, which give the SNR a scalloped appearance. Many of the filaments appear to be unresolved $\left(<6^{\prime \prime}\right)$ in width. There are only a few locations where the emission is better characterized as unresolved knots or clusters of knots. The BEK is resolved into several intersecting arced filaments and diffuse emission. This detail is shown in Figure 3 and identified with the nomenclature used by Hwang et al. (2005). These structures do not appear very different from the rest of the SNR except for being several times brighter and marking a distinct indentation in the periphery of the SNR.

At $160 \mu \mathrm{m}$ emission is not evident from the SNR, including the BEK. Extrapolations of IRS spectral fits (Section 3.2.2) predict $160 \mu \mathrm{m}$ brightnesses of $\sim 6-12 \mathrm{MJy} \mathrm{sr}^{-1}$ for the BEK. The mean intensity of the $160 \mu \mathrm{m}$ cirrus is $\sim 130 \mathrm{MJy} \mathrm{sr}^{-1}$ with an rms variation of $\sim 15-20 \mathrm{MJy} \mathrm{sr}^{-1}$. Thus, as most of Puppis $\mathrm{A}$ is many times fainter than the BEK, it is no surprise that the SNR is not visible at $160 \mu \mathrm{m}$.

Comparison with X-ray images in Figures 2 and 3 shows a strong correlation between IR and X-ray emission on all spatial scales down to the $\sim 6^{\prime \prime}$ resolution of the $24 \mu \mathrm{m}$ MIPS data. In comparison, the correlation with the radio emission (Castelletti et al. 2006) is relatively poor, especially on small spatial scales. The distribution of optical filaments and knots in Puppis A is very different from that of the IR, X-ray, or radio emission. Indeed, only some of the brightest optical features have detectable $24 \mu \mathrm{m}$ counterparts. The most notable of these is the O-rich ejecta of the "Omega Filament," which is the only optical filament to have a bright easily distinguished $24 \mu \mathrm{m}$ counterpart $(\alpha, \delta)=\left(8^{\mathrm{h}} 22^{\mathrm{m}} .8,-42^{\circ} 46^{\prime} \cdot 6\right)$. The adjacent X-ray emitting ejecta observed by Katsuda et al. (2008) also has highly correlated $24 \mu \mathrm{m}$ emission, but it is three times fainter than that of the Omega Filament.

The IRAC imaging only covered the region of the BEK. Emission associated with the BEK is very faint and confused. Therefore the IRAC images shown in Figure 3 have had stellar sources removed by subtraction of scaled versions of the $4.5 \mu \mathrm{m}$ image for the $8 \mu \mathrm{m}$ data and scaled versions of the $3.6 \mu \mathrm{m}$ image for the 5.8 and $4.5 \mu \mathrm{m}$ data. This subtracts stellar emission which is relatively blue in color, with minimal alteration of the relatively red emission of the BEK and the ISM. At $3.6 \mu \mathrm{m}$ such a template subtraction is not possible, so the version shown is generated from the point source fitting and subtraction when processing the BCD data with MOPEX/APEX (Makovoz $\&$ Marleau 2005). The BEK is most clearly seen at $8 \mu \mathrm{m}$ where emission lies along the leading (eastward) edges of the brightest $24 \mu \mathrm{m}$ features (the "Compact Knot" and the "Bar"). The peak intensity is $I(8 \mu \mathrm{m})<0.3 \mathrm{MJy} \mathrm{sr}^{-1}$. The ratio $I(8 \mu \mathrm{m}) / I(5.8 \mu \mathrm{m})=2.7$. The ratios $I(8 \mu \mathrm{m}) / I(4.5 \mu \mathrm{m})$ and $I(8 \mu \mathrm{m}) / I(3.6 \mu \mathrm{m})$ appear to be $\sim 10$. These IRAC colors are consistent with polycyclic aromatic hydrocarbon (PAH) emission as seen at other locations in the ISM, e.g., in the direction of the Galactic center (Arendt et al. 2008).

\subsubsection{Global Properties}

The MIPS observations cover all of Puppis A and hence we used them to measure the total flux density of the SNR at 24 


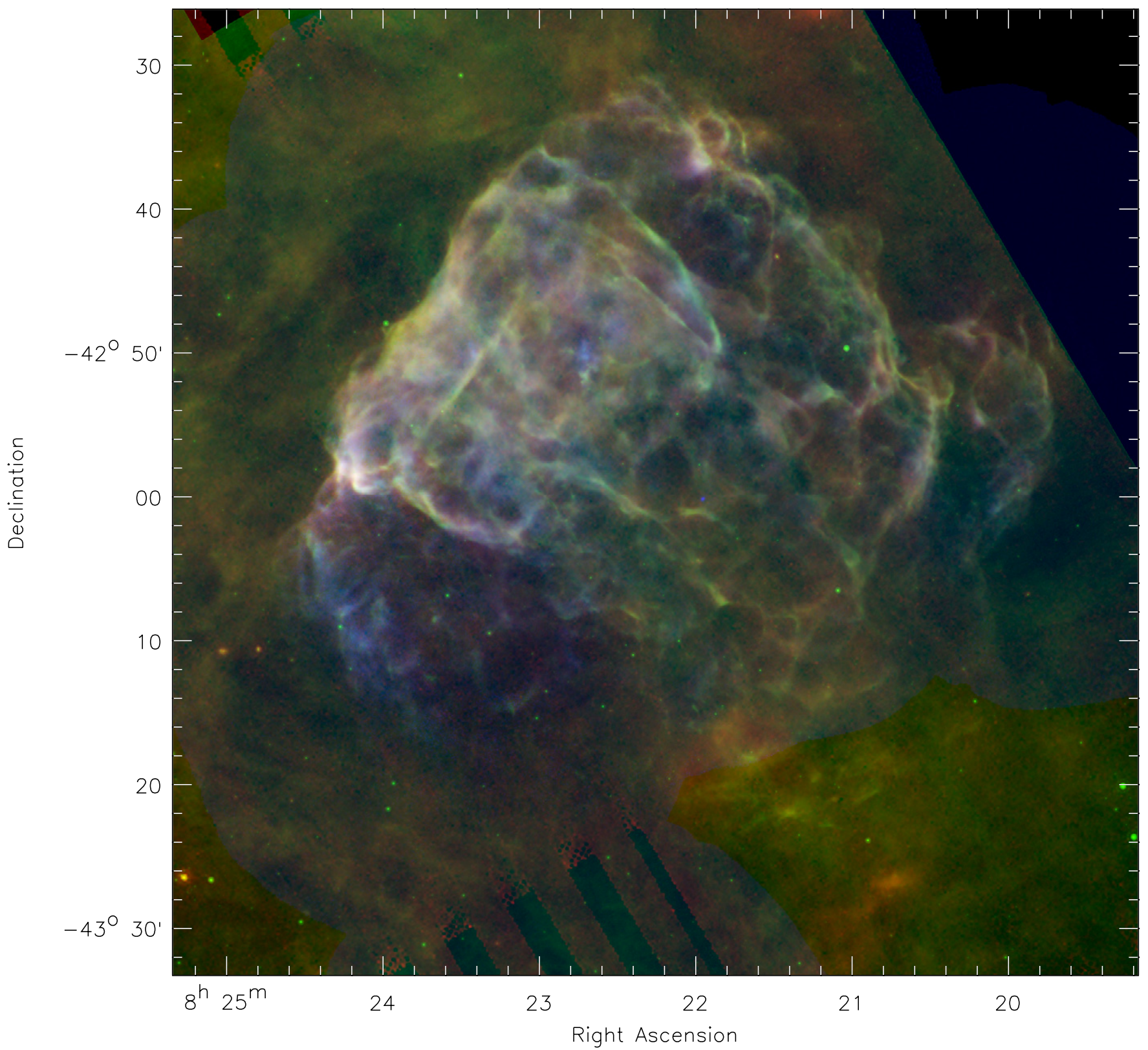

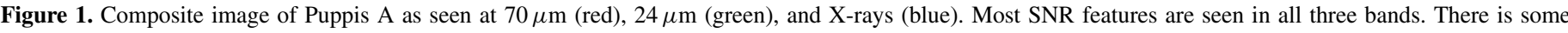
intrinsic variation in the relative colors, but structure in the $70 \mu \mathrm{m}$ background also leads to some apparent color features.

and $70 \mu \mathrm{m}$, the main uncertainty being confusion from ISM along the line of sight. To minimize this problem, we used the $160 \mu \mathrm{m}$ image, where we do not see the SNR, as a template for the ISM emission. This was scaled and subtracted from the 24 and $70 \mu \mathrm{m}$ images. The resulting maps had a smoother flatter background with a mean value near 0 . The SNR intensity was integrated within an irregularly shaped aperture that was defined jointly by surface brightness thresholds of the 24 and $70 \mu \mathrm{m}$ images. An expanded annular aperture around this region was used to fine-tune the local background level at the SNR. The integrated flux changed by $\sim 5 \%$ depending on the width of the annular aperture used to set the local background. This was used as the quoted uncertainty of the integrated flux densities. Two upper limits for the $160 \mu \mathrm{m}$ flux density were measured. The first simply integrated all the emission within the area of the SNR after subtraction of a flat background at the local minimum intensity $\left(90 \mathrm{MJy} \mathrm{sr}^{-1}\right)$. This limit includes much of the ISM along the line of sight. A more restrictive limit was obtained by assuming that the $160 \mu \mathrm{m}$ emission is spatially correlated with the 24 and $70 \mu \mathrm{m}$ emission. The slopes of the linear correlations between the $160 \mu \mathrm{m}$ intensity and the 24 and $70 \mu \mathrm{m}$ intensities were used to scale the shorter wavelength flux densities to a $160 \mu \mathrm{m}$ flux density. The resulting flux densities are listed in Table 1 . The results are very similar to the IRAS measurement of Arendt et al. (1991), but with smaller uncertainties resulting from the better spatial resolution and the use of the $160 \mu \mathrm{m}$ data to reduce uncertainties associated with the line-of-sight ISM.

\subsection{Spectra and Models}

\subsubsection{The East Cloud}

The Spitzer IRS spectra of the East Cloud are important to this study in that they allow us to characterize the composition and 


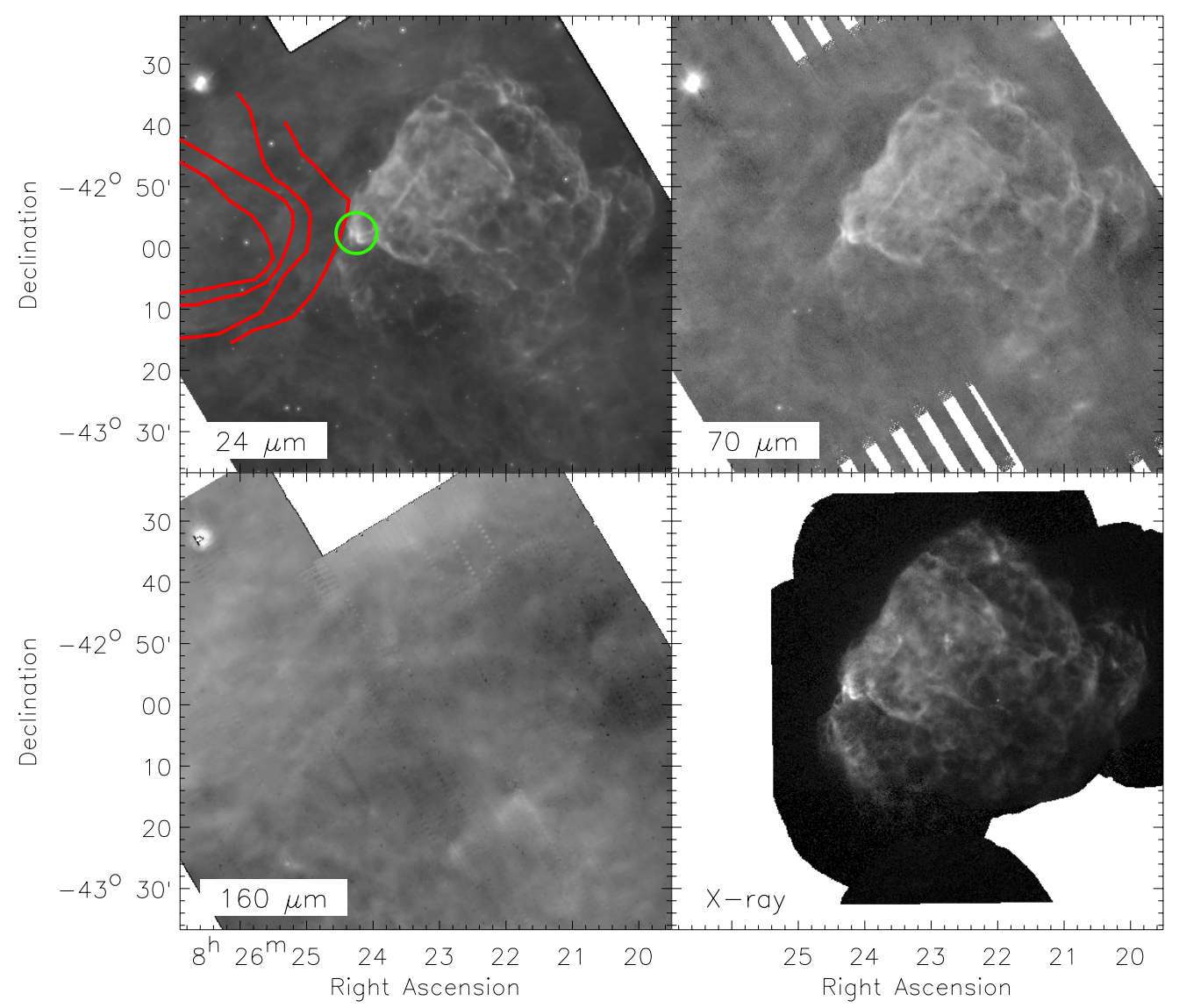

Figure 2. Spitzer MIPS images of the Puppis A SNR and the surrounding ISM at 24, 70, and $160 \mu \mathrm{m}$. The ROSAT HRI image is shown in the lower right panel. On the $24 \mu \mathrm{m}$ image, contours of CO emission (12-17 km s${ }^{-1}$ ) of the East Cloud (Dubner \& Arnal 1988) are superimposed, and a circle marks the BEK where the SNR interacts with outlying clumps of this cloud. At 24 and $70 \mu \mathrm{m}$, IR emission of the SNR is well correlated with the X-ray emission, but at $160 \mu \mathrm{m}$ it is lost in the confusion of the ISM.

(A color version of this figure is available in the online journal.)

Table 1

Integrated Flux Density of Puppis A

\begin{tabular}{lcc}
\hline $\begin{array}{c}\text { Wavelength } \\
(\mu \mathrm{m})\end{array}$ & $\begin{array}{c}\text { Flux Density }^{\mathrm{a}} \\
(\mathrm{Jy})\end{array}$ & Satellite \\
\hline 12 & $<100$ & IRAS \\
24 & $230 \pm 11$ & Spitzer \\
25 & $245 \pm 120$ & IRAS \\
60 & $1195 \pm 350$ & IRAS \\
70 & $1025 \pm 51$ & Spitzer \\
100 & $1265 \pm 990$ & IRAS \\
160 & $<330^{\mathrm{b}}$ & Spitzer \\
160 & $<6000^{\mathrm{c}}$ & Spitzer \\
\hline
\end{tabular}

Notes.

${ }^{a}$ IRAS results are from Arendt et al. (1991) and Spitzer results are from the present work.

${ }^{\mathrm{b}}$ Limit for emission that is spatially correlated with 24 and $70 \mu \mathrm{m}$ emission.

${ }^{\mathrm{c}}$ Limit for all emission across the extent of the SNR.

grain size distribution of the interstellar dust within a cloud that is physically associated with the SNR. This provides a direct measure of the state of the dust prior to its processing by the SNR shock. For most other SNRs, the nature of the interstellar dust must be assumed or simply measured only as a line-of-sight integral.

The primary location for the East Cloud spectrum was selected near the brightest portion of the cloud, near $(\alpha, \delta)=$ $\left(8^{\mathrm{h}} 26^{\mathrm{m}} 50^{\mathrm{s}} .50,-42^{\circ} 55^{\prime} 26^{\prime \prime} .0\right)$, as indicated by the IRAS $25 \mu \mathrm{m}$ images. (The slit locations are indicated in Figure 4.) The SL and LL spectral extractions are necessarily over different regions due to the slit sizes and orientations. To maximize the signal-tonoise ratio, we extracted the average spectrum over the entire subslit for each spectral order. The LL and SL spectral orders are normalized such that the regions of spectral overlap coincide in intensity. The resulting spectrum is shown in Figure 5(a). At these wavelengths, the East Cloud spectrum is dominated by the typical PAH emission bands. The $\mathrm{H}_{2} \mathrm{O}-0 S(0)$ and $S(1)$ rotational lines are detected at 28.2 and $17.0 \mu \mathrm{m}$. Thermal emission from larger dust grains is of increasing significance at $\lambda \gtrsim 20 \mu \mathrm{m}$.

We fit these spectra using a Zubko et al. (2004; hereafter ZDA) BARE-GR-FG model, radiatively heated by an ambient interstellar radiation field (ISRF). The ZDA models are a set of models for the dust grain size (or mass) distributions that are derived to simultaneously fit average interstellar extinction, emission, and abundances. Different models are distinguished by different choices of grain compositions and different sets of abundance constraints. The BARE-GR-FG model comprises PAHs, silicate, and graphite grains without any mantles and is fit to elemental abundances determined from $F$ and $G$ stars (Sofia \& Meyer 2001). The BARE-GR-FG model is the best of the ZDA bare gain models. The primary free parameter of our fit to the East Cloud spectrum is the strength of the ISRF, $\chi=I_{\text {East Cloud }} / I_{\text {local }}$, which was varied over the range $\log (\chi)=[-2,+3]$ in steps of 0.5 , with $I_{\text {local }}$ defined by the local ISRF as characterized by Mathis et al. (1983). The overall 


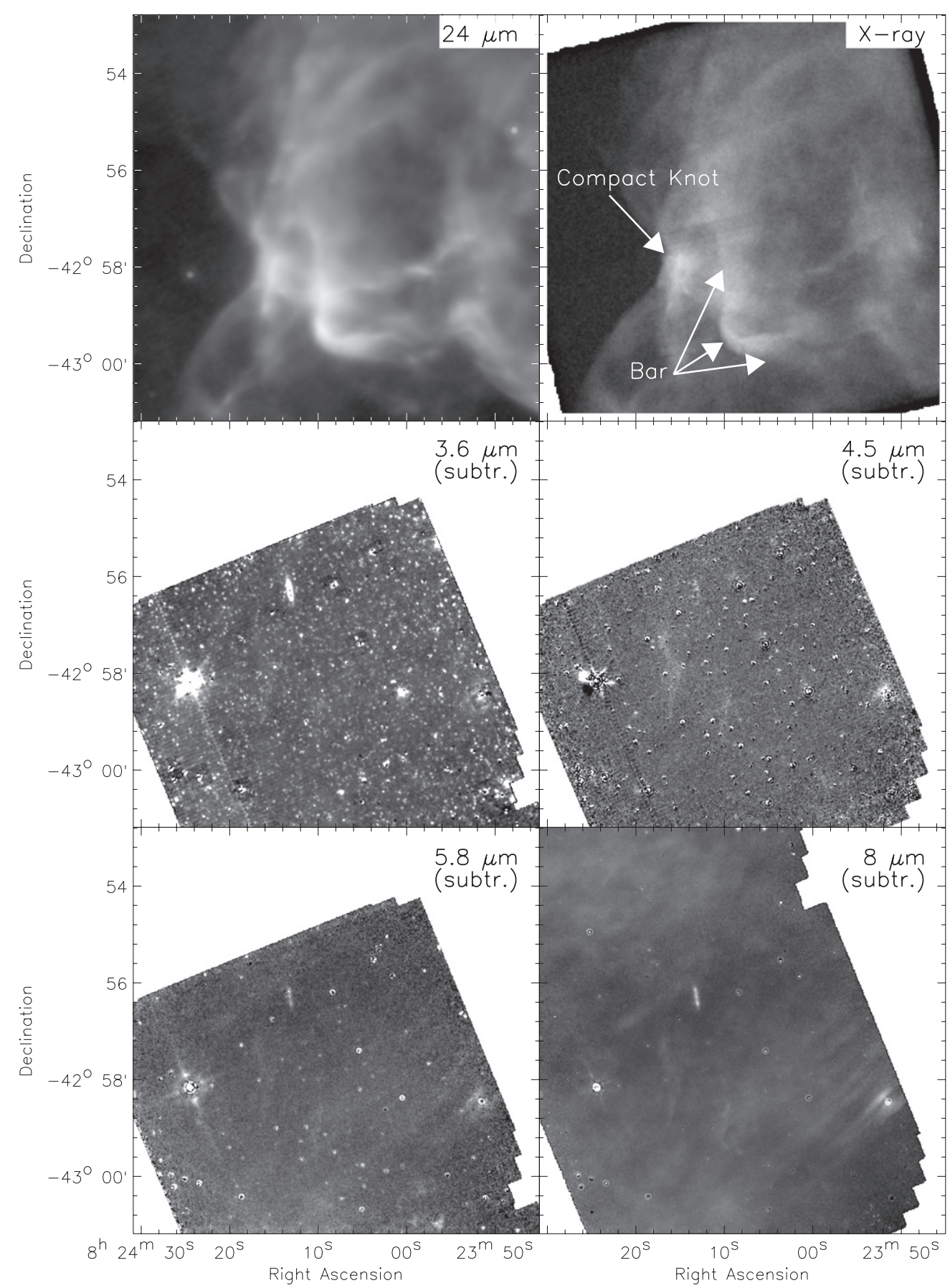

Figure 3. Top panels show the full resolution $24 \mu \mathrm{m}$ image and the Chandra ACIS image at the location of the BEK. The other panels show the corresponding Spitzer IRAC images. The stellar sources are subtracted to emphasize the faint diffuse emission. (See the text for details.) The Compact Knot and Bar are both detected, but are faint and confused at $3.6-8 \mu \mathrm{m}$.

normalization of the spectrum (proportional to the column density of the emitting material) is the second free parameter allowed in the fitting. The nominal best fit is found for a moderately enhanced ISRF, $\log (\chi)=1$, and is shown in Figure 5(a). The enhancement could be related to emission from the SNR itself, similar to the precursor observed outside of SNR N132D (Morse et al. 1996). However, the spectrum is at a location that is $\sim 20 \mathrm{pc}$ (in projection) from the nearest edge of the SNR and there is no indication of a gradient in the ISRF with respect to distance from the SNR.

The errors in the fit shown in Figure 5(a) appear to be systematic within the different spectral orders in which the data are obtained. This suggests that the data in the different spectral orders may not be accurately normalized. Therefore, we applied an alternate strategy of normalizing each spectral order of the data independently to optimally match the model(s). The switch from 1 to 4 normalization parameters does not provide any additional physical significance, but it does permit compensation for systematic errors between the data in different spectral orders. This strategy again finds a best fit for $\log (\chi)=1$ as shown in Figure 5(b). In this case, the normalizations used for the spectral orders [SL1, SL1, LL2, LL1] are [0.63, 0.72, $1.35,0.82]$ times those used to normalize the orders by their overlap. As a check on this procedure, we similarly fit the 

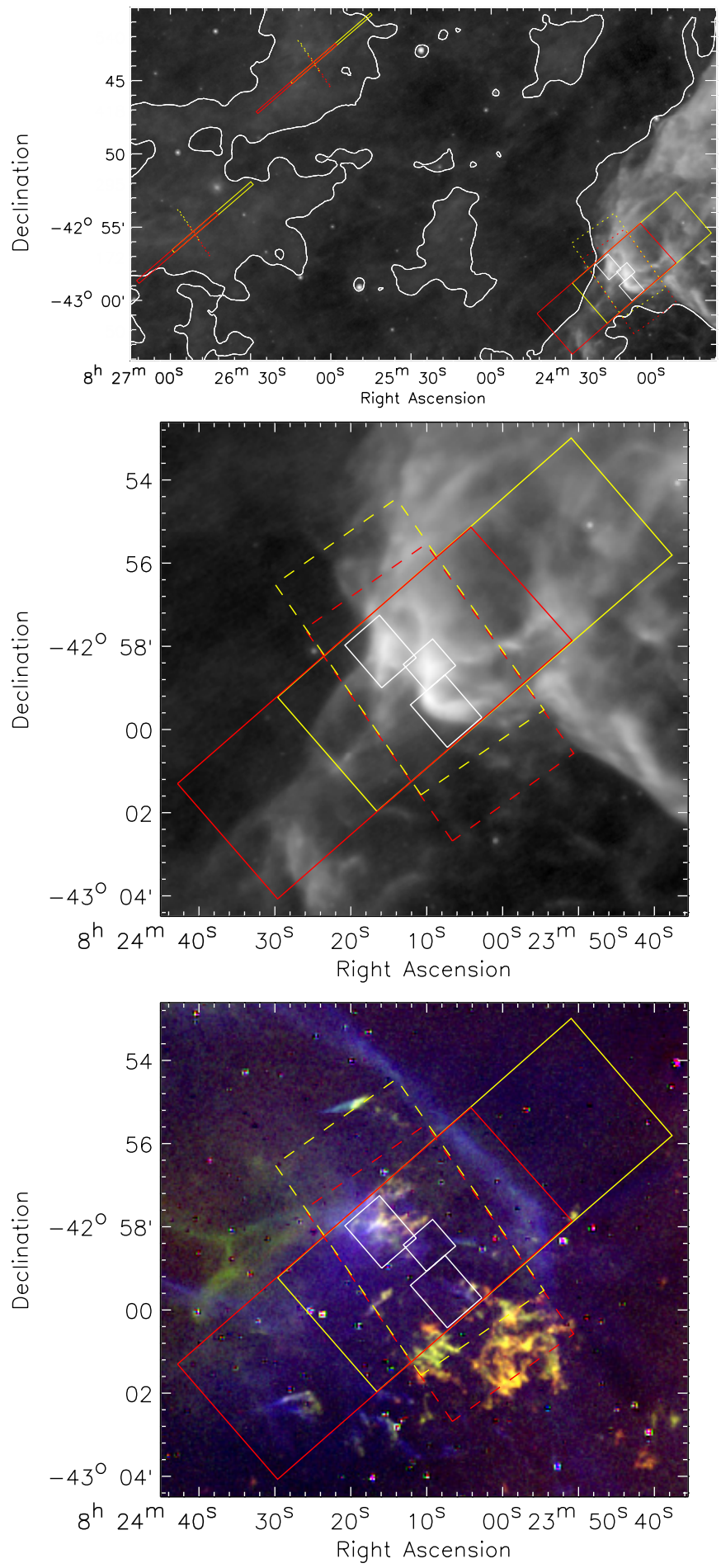

Figure 4. Top: wide view of the IRS coverage showing the BEK and the two pointings at the brightest part of the East Cloud and a northern extension of the cloud. A single contour at $20.5 \mathrm{MJy} \mathrm{sr}^{-1}$ helps delineate the extent of the East Cloud. Middle: IRS coverage of the BEK superimposed on the $24 \mu \mathrm{m}$ MIPS image. The solid red and yellow lines indicate the regions of the fully sampled LL1 $(19.5-38.0 \mu \mathrm{m})$ and LL2 $(14.0-21.3 \mu \mathrm{m})$ spectral maps, respectively. The dashed red and yellow lines indicate the regions of the undersampled SL1 $(7.4-14.5 \mu \mathrm{m})$ and SL2 $(5.2-7.7 \mu \mathrm{m})$ spectral maps, respectively. The white boxes indicate the regions where we have extracted mean spectra for a detailed analysis. From northeast to southwest these are designated: "Compact Knot," "Bar-N," and "Bar-S." Bottom: the same coverage and analysis regions are superimposed on an optical emission line image- $\mathrm{H} \alpha$ : red; [S II]: green; [O III]: blue. Only the Compact Knot region contains strong line emission. spectrum at a second location, in a northern extension of the East Cloud at $(\alpha, \delta)=\left(8^{\mathrm{h}} 26^{\mathrm{m}} 04^{\mathrm{s}} .60,-42^{\circ} 44^{\prime} 01^{\prime \prime} .6\right)$. These data, while slightly fainter, are a slightly better match to the ZDA model (Figure 5(c)), again with $\log (\chi)=1.0$.

These results show that the ZDA BARE-GR-FG model provides a good representation of the dust giving rise to the emission of the East Cloud. The results indicate a moderate $(\sim 10 \times)$ enhancement in the strength of the ISRF in the region. This is largely a result of the rising intensity at wavelengths $>20 \mu \mathrm{m}$. As the ISRF strength increases, large dust grains are driven to higher temperatures and the slope of this rise increases. The most significant discrepancy between the data and the model is in the region of the LL2 spectral order, 14-21 $\mu \mathrm{m}$. In this spectral region, it appears that the ZDA model's absorption coefficients (most likely for the PAHs) are roughly a factor of two too large (as implied by Figure 5(a)). An alternate possibility is that the model is a very good match, and there are systematic errors in the data that cause apparent discontinuities at the ends of the SL1, LL2, and/or LL1 spectral orders (as shown in Figures 5(b) and (c)).

\subsubsection{The Bright Eastern Knot}

Having demonstrated that the ZDA dust model provides a reasonable fit to the East Cloud, we now apply the same model to the spectra of the three brightest portions of the BEK. The selected regions are indicated in Figure 4. The easternmost region corresponds to the "Compact Knot" feature of Hwang et al. (2005), while the other two cover the "Bar" region. We designate these latter two regions as "Bar-N" and "Bar-S." The spectra are shown in Figure 6. These spectra are very different from those of the East Cloud: the strong PAH features are absent, and the peak of the thermal emission of the large grains is shifted from $\sim 100 \mu \mathrm{m}$ to $\sim 50 \mu \mathrm{m}$.

For the spectra shown in Figure 6, atomic line emission is only evident in the compact knot region. Examination of the spectral data cubes and emission line maps (Figure 7) reveals that the line emission is not correlated with the dust. Instead the lines are correlated with optical knots and filaments scattered in the vicinity of the BEK. Lines of singly ionized species ([Ne II] $12.8 \mu \mathrm{m}$ and [Fe II] 17.9 and $26.0 \mu \mathrm{m}$ ) are correlated with optical $\mathrm{H} \alpha$ and [S II] emission, while more highly ionized species ([O IV] $26.0 \mu \mathrm{m}$, [Ne III] $15.6 \mu \mathrm{m}$, and [S III] 18.7 and $33.5 \mu \mathrm{m}$ ) are correlated with optical [O III] emission. (We note that part of the optical line emission, especially the long linear [O III] structures in Figure 7, is part of the Vela SNR which lies in the foreground of Puppis A. Despite the superposition of the two SNRs, we see no evidence for any IR line or continuum emission from the Vela SNR that would affect the analysis of Puppis A.) No molecular $\mathrm{H}_{2}$ emission lines are observed in these background-subtracted spectra, although line-of-sight $\mathrm{H}_{2}$ emission is fairly uniform over the entire spectral map prior to the background subtraction. This lack of $\mathrm{H}_{2}$ is consistent with results from Paron et al. (2008) who find no ${ }^{12} \mathrm{CO}$ emission directly associated with the BEK, despite its presence in more distant portions of the East Cloud and even in small clumps that appear unshocked and in the foreground to the BEK. They conclude that either the gas currently being swept up is not part of a molecular clump or the molecular gas is being dissociated by a radiative precursor.

The interstellar dust that has been swept up into the SNR resides in a vastly different environment than the general ISM. 


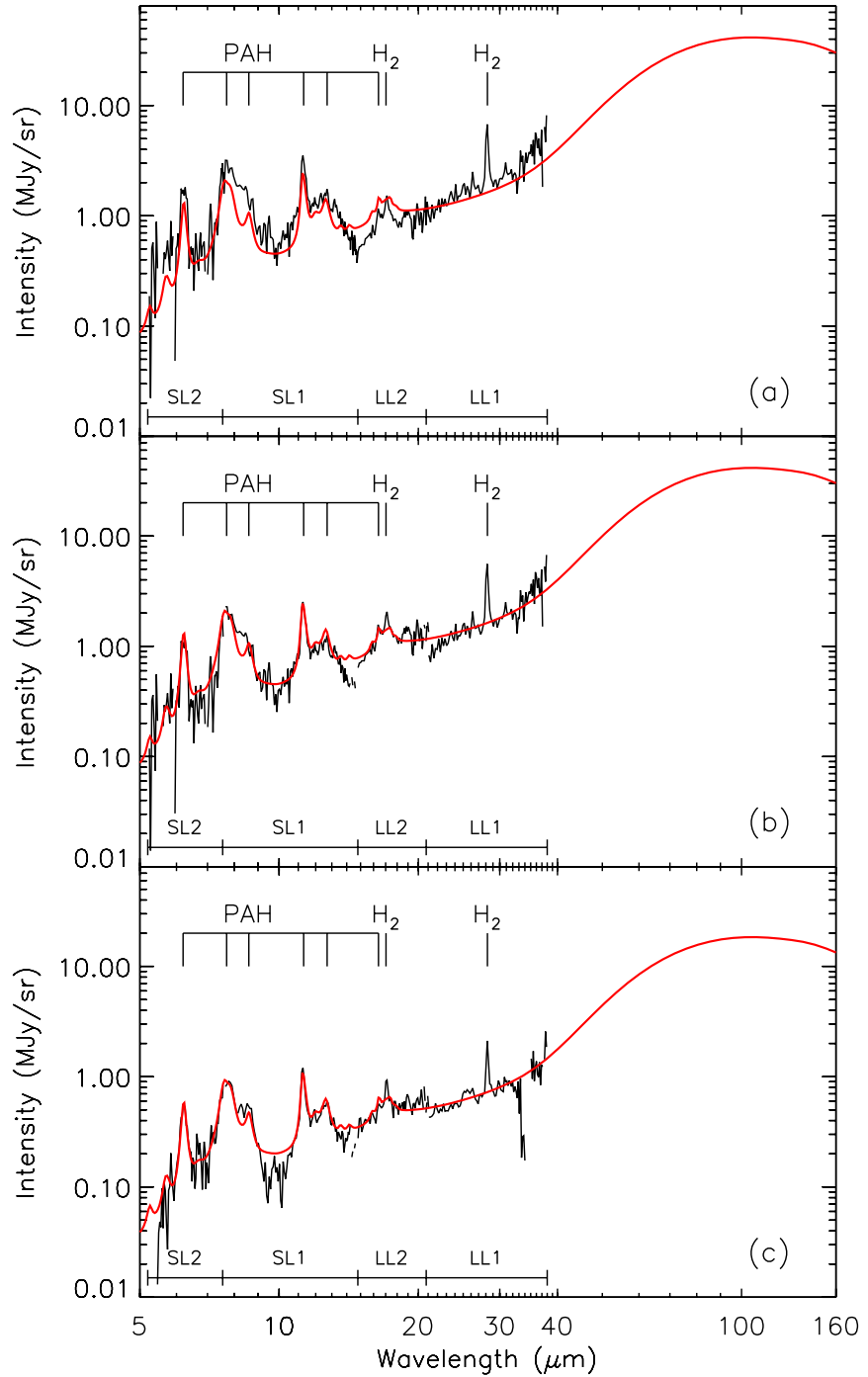

Figure 5. (a) ZDA model (red line) fit to the spectrum of the East Cloud (b) Spectrum of the East Cloud fit to the ZDA model, allowing independent normalization of each spectral order (labelled along the bottom of the plot). (c) Spectrum of the East Cloud at the second (northern) location fit to the ZDA model, allowing independent normalization of each spectral order.

(A color version of this figure is available in the online journal.)

The strong correlation of the 24 and $70 \mu \mathrm{m}$ emission with the $\mathrm{X}$-ray emission indicates that the dust is located within the shocked X-ray emitting gas. In such an environment, collisions with electrons are the dominant heating source for the dust, even if the local radiation field is elevated by factors of 10 as indicated by the East Cloud. Thus, instead of the radiation field strength, the free parameters of the dust model are the electron density, $n_{e}$, and temperature, $T_{e}$, of the X-ray emitting gas. A third free parameter characterizes the amount of grain destruction (if any) that has taken place via thermal sputtering of the dust population. Independent of the grain radius, $a$, sputtering (mainly by protons) acts to reduce the size of the grains by an amount $\Delta a \sim n_{H} t \sim n_{e} t$ where $t$ is the amount of time the dust has been subjected to the sputtering.

As with the East Cloud, we calculated a grid of models, now involving a three-dimensional parameter space, $\left\{n_{e}, T_{e}, \Delta a\right\}$, instead of only one dimension $\{\chi\}$. The gas parameters are varied logarithmically over the range of $\log _{2}\left[n_{e}\left(\mathrm{~cm}^{-3}\right)\right]=[0,5]$ in ten steps of 0.5 , and $\log \left[T_{e}(\mathrm{~K})\right]=[5.75,7.25]$ in seven steps

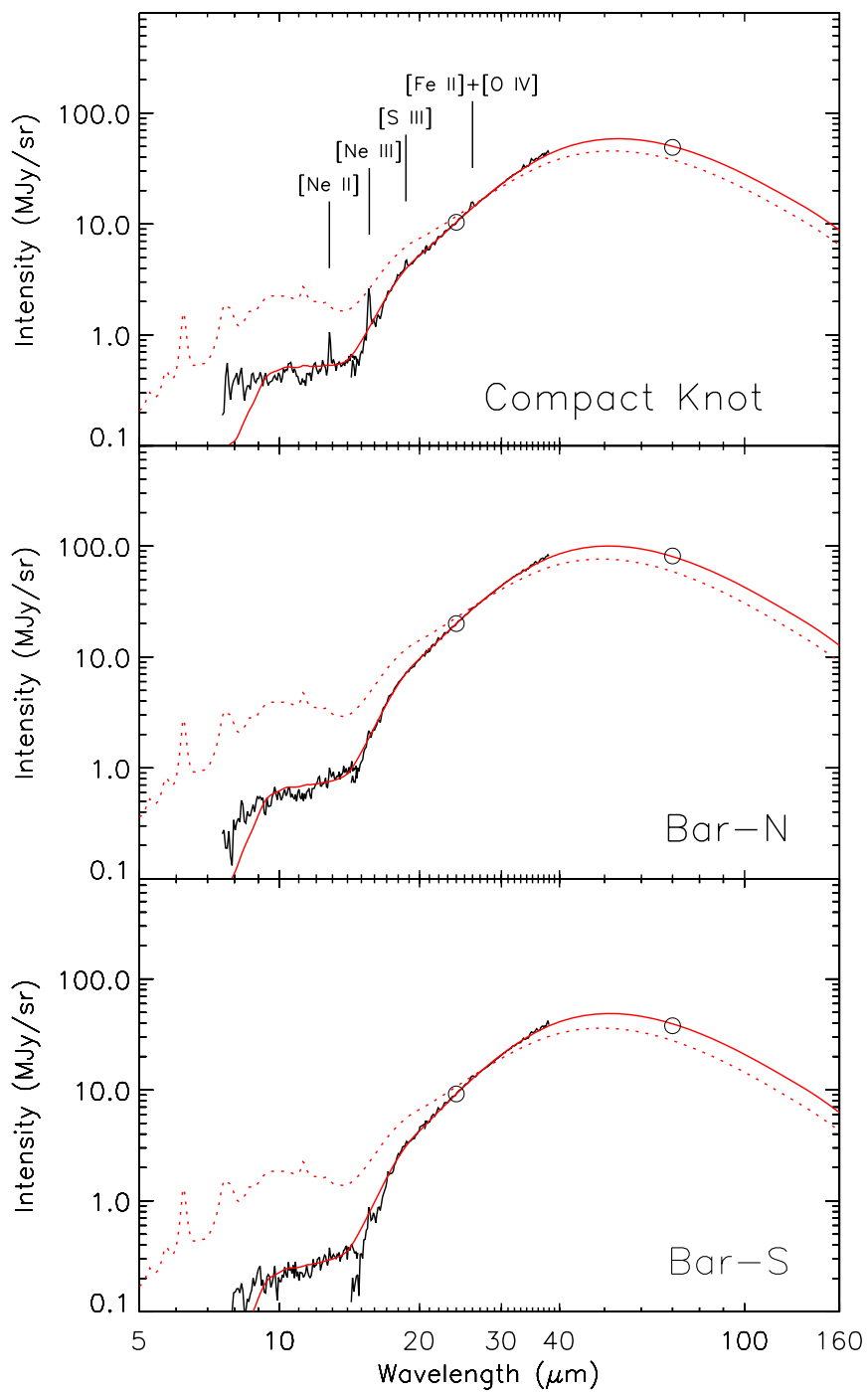

Figure 6. Spectra of the BEK regions fit to the ZDA model. The solid red lines show the best fitting models. The dotted red lines show models for the same gas parameters $\left(T_{e}, n_{e}\right)$ but with no sputtering $(\Delta a=0)$. The circles indicate the MIPS measurements at 24 and $70 \mu \mathrm{m}$. These are normalized to match the IRS data at $24 \mu \mathrm{m}$. The $70 \mu \mathrm{m}$ data are well fit by the models even though the models were fit only to the IRS data.

(A color version of this figure is available in the online journal.)

of 0.25 ; i.e., $1<n_{e}<32 \mathrm{~cm}^{-3}$ and $0.56 \times 10^{6}<T_{e}<$ $17.8 \times 10^{6} \mathrm{~K}$. We varied the sputtering linearly over the range of $\Delta a=[0,0.01] \mu \mathrm{m}$ in steps of $0.0005 \mu \mathrm{m}=5 \AA$. We apply the same $\Delta a$ for all grain compositions. This results in a grid of $10 \times 7 \times 21$ models.

Again, each spectral order was normalized to fit each model in the grid to determine the single best-fitting (minimum $\chi^{2}$ ) model. However, the SL2 data $(5-7.7 \mu \mathrm{m})$ were not included due to a lack of any detectable signal. Additionally, the SL1 data $(7.4-14.5 \mu \mathrm{m})$ showed evidence of an oversubtracted background: there was a strong negative feature at the location of the $11.3 \mu \mathrm{m}$ PAH band, and the spectra show a steady slope through zero and into negative intensities. This cannot be easily alleviated by selecting a different background region because the large-scale gradient in ISM intensity (seen best at $160 \mu \mathrm{m}$, Figure 2) and small-scale local variations happen to place the BEK in a low-intensity region relative to the available off-source background pointings (better illustrated 
Table 2

IR Spectral Model Parameters for BEK Regions

\begin{tabular}{lcccccc}
\hline \hline \multicolumn{1}{c}{ Region } & $\Delta a(\mu \mathrm{m})$ & $n_{e}\left(\mathrm{~cm}^{-3}\right)$ & $T_{e}(\mathrm{~K})$ & $\chi_{v}^{2}$ & \multicolumn{2}{c}{ X-ray } \\
\cline { 4 - 7 } & & & & & $n_{e} t\left(\mathrm{~cm}^{-3} \mathrm{~s}\right)$ & $T_{e}(\mathrm{~K})$ \\
\hline Compact Knot & 0.0015 & 4.0 & $3.2 \times 10^{6}$ & 2.79 & $1.70 \times 10^{11}$ & $5.8 \times 10^{6}$ \\
Bar-N & 0.0020 & 5.7 & $3.2 \times 10^{6}$ & 2.88 & $2.95 \times 10^{11}$ & $7.1 \times 10^{6}$ \\
Bar-S & 0.0025 & 5.7 & $3.2 \times 10^{6}$ & 9.56 & $2.77 \times 10^{11}$ & $7.4 \times 10^{6}$ \\
\hline
\end{tabular}

at 24 and $70 \mu \mathrm{m}$, Figure 2). Therefore, rather than fitting with just a single normalization coefficient, $I_{\text {data }}=A I_{\text {model }}$, the fit involved determining the coefficients $A, B$, and $C$ in $I_{\text {data }}=A I_{\text {model }}+B I_{\mathrm{ISM}}+C$. The new $B$ coefficient directly accounts for oversubtraction of the ISM background ( $I_{\text {ISM }}$ is given by the model fit to the East Cloud), and the new $C$ term allows for a more generic, constant error in the background.

The fitted spectra for the three BEK regions are shown in Figure 6. In the figure, we have also overplotted the mean intensities for these regions as extracted from the MIPS 24 and $70 \mu \mathrm{m}$ images (open circles). These MIPS intensities were normalized to match the IRS data (not the model) at $24 \mu \mathrm{m}$, but were not used as a constraint in fitting the models. The corresponding $n_{e}, T_{e}$, and $\Delta a$ parameters are listed in Table 2, along with the reduced $\chi_{v}^{2}$ values of the fits. For comparison, the mean gas parameters derived from modeling the X-ray spectra (Hwang et al. 2005) are also listed. The IRS spectra of all three regions are dominated by continuum emission from dust that is much warmer than the typical ISM. Larger $0.1 \mu \mathrm{m}$ grains are at equilibrium temperatures of $T \sim 48 \mathrm{~K}$, while smaller gains are radiating at higher (and fluctuating) temperatures. Thus, the resulting spectra are warmer and not well characterized by a single temperature. PAH emission is not evident in the spectra, despite the apparent presence of weak PAH emission in the IRAC imaging. The IRAC intensities indicate that the PAH emission is $<0.3 \mathrm{MJy} \mathrm{sr}^{-1}$, which would be lost in the noise and systematic errors of background subtraction in the IRS spectra. Figure 6 also shows model spectra for the best fit $n_{e}$ and $T_{e}$, but with the requirement that no grains were destroyed, i.e., $\Delta a=0.0$. These model spectra (dotted lines) are based on the same dust content as the ISM, but the collisional heating within the SNR raises the grain temperatures which reduces the relative strength of the PAH features (cf. Figure 5). The excessive emission at short wavelengths is a result of excluding dust sputtering in these models.

Figure 8 shows two-dimensional slices through the threedimensional $\chi_{v}^{2}$ parameter space at the location of minimum $\chi_{v}^{2}$. Models with $\chi_{v}^{2} \lesssim 2 \min \left(\chi_{v}^{2}\right)$ are reasonable fits to the data. The grid of models used was fairly coarse, but the broadness of the $\chi_{v}^{2}$ contours in these slices indicates that higher precision is not demanded. The slices demonstrate two fundamental correlations of the parameters of the fits. The first is that the heating rate of a dust grain is proportional to $n_{e} T_{e}^{3 / 2}$ when the electrons are completely stopped by the grains (Dwek et al. 2008). This dependence is the product of the collision rate $\sim n_{e} v_{e}$ (where $v_{e} \sim\left(k T_{e}\right)^{1 / 2}$ ) and the kinetic energy of the electrons $\sim k T_{e}$. This is the major trend in the left column panels in Figure 8 and is shown as a dotted line. The spectrum of a large grain at its equilibrium temperature will depend on $n_{e} T_{e}^{3 / 2}$, and $n_{e}$ and $T_{e}$ cannot be determined independently. However, in practice, this degeneracy is broken and a minimum in $\chi_{v}^{2}$ is localized because the models (and the SNR) include a range of grain sizes, and only the largest grains reside at equilibrium temperatures determined strictly by $n_{e} T^{3 / 2}$. Smaller grains have fluctuating temperatures and may not completely stop the electrons. Because of these effects, the spectrum is sensitive to the grain size distribution as well as the gas temperature and density. The second correlation is the inverse relationship between the electron density $\left(n_{e}\right)$ and the amount of sputtering $(\Delta a)$, as illustrated in the right column panels of Figure 8. Increases in electron density raise the heating rate and thus the grain temperature, which produces more short wavelength emission. Conversely, increasing the amount of sputtering reduces the abundance of the smallest grains in the size distribution, leading to a decrease in the short wavelength emission because the small grains are hotter than the larger grains. The center column panels of Figure 8 exhibit a superposition of these two fundamental trends.

\subsubsection{The Total SNR}

As a demonstration of the usefulness of the spectroscopic data versus SEDs constructed from broadband imaging, we have also fit the SED of the entire SNR (Table 1) using the grid of collisionally heated dust models. Figure 9 shows the results. The fits are almost entirely constrained by the 24 and $70 \mu \mathrm{m}$ MIPS data points, with their smaller uncertainties. There is a wide range of models that can fit the observed 24 to $70 \mu \mathrm{m}$ color and the IRAS data points as well. Acceptable models can be found up to the tested limits (and presumably beyond) of the $\left\{T_{e}, n_{e}, \Delta a\right\}$ parameter space. The lack of useful measurements at $\lambda<24 \mu \mathrm{m}$ or any constraint on the slope of the spectrum at $24 \mu \mathrm{m}$ makes it impossible to assess the relative abundance of small grains and the amount of sputtering. If we assume that there is no separate cooler component of the dust that is not correlated with the 24 and $70 \mu \mathrm{m}$ emission (i.e., adopt the lower of the $160 \mu \mathrm{m}$ upper limits), then the integrated IR flux of the SNR is $9.0 \times 10^{-8} \mathrm{erg} \mathrm{s}^{-1} \mathrm{~cm}^{-2}$, corresponding to a luminosity of $1.4 \times 10^{4} L_{\odot}$ and a dust mass of $0.25 M_{\odot}$ at a distance of $2.2 \mathrm{kpc}$. The luminosity and mass are in very good agreement with those previously reported (Arendt et al. 1991), although the flux is 1.32 times higher than reported by Dwek et al. (1987). Accordingly, the ratio of IR to X-ray emission of Puppis A is also higher than that of Dwek et al. (1987), with IRX $=F_{\mathrm{IR}} / F_{0.2-4.0 \mathrm{keV}}=6.9$, which is still a factor of $\sim 5$ below the theoretically expected ratio. This factor is too large to attribute to the grain destruction by sputtering and must represent a relatively low dust-to-gas mass ratio in material being swept up as concluded by Dwek et al. (1987).

\section{DISCUSSION}

\subsection{Grain Destruction in Shocks}

The excellent correlation between IR and X-ray emission revealed by the high-resolution MIPS imaging strengthens the direct association of the dust with the X-ray emitting gas, which had been indicated by the IRAS data (Arendt et al. 1991). Puppis A had not been observed with the Infrared Space Observatory 


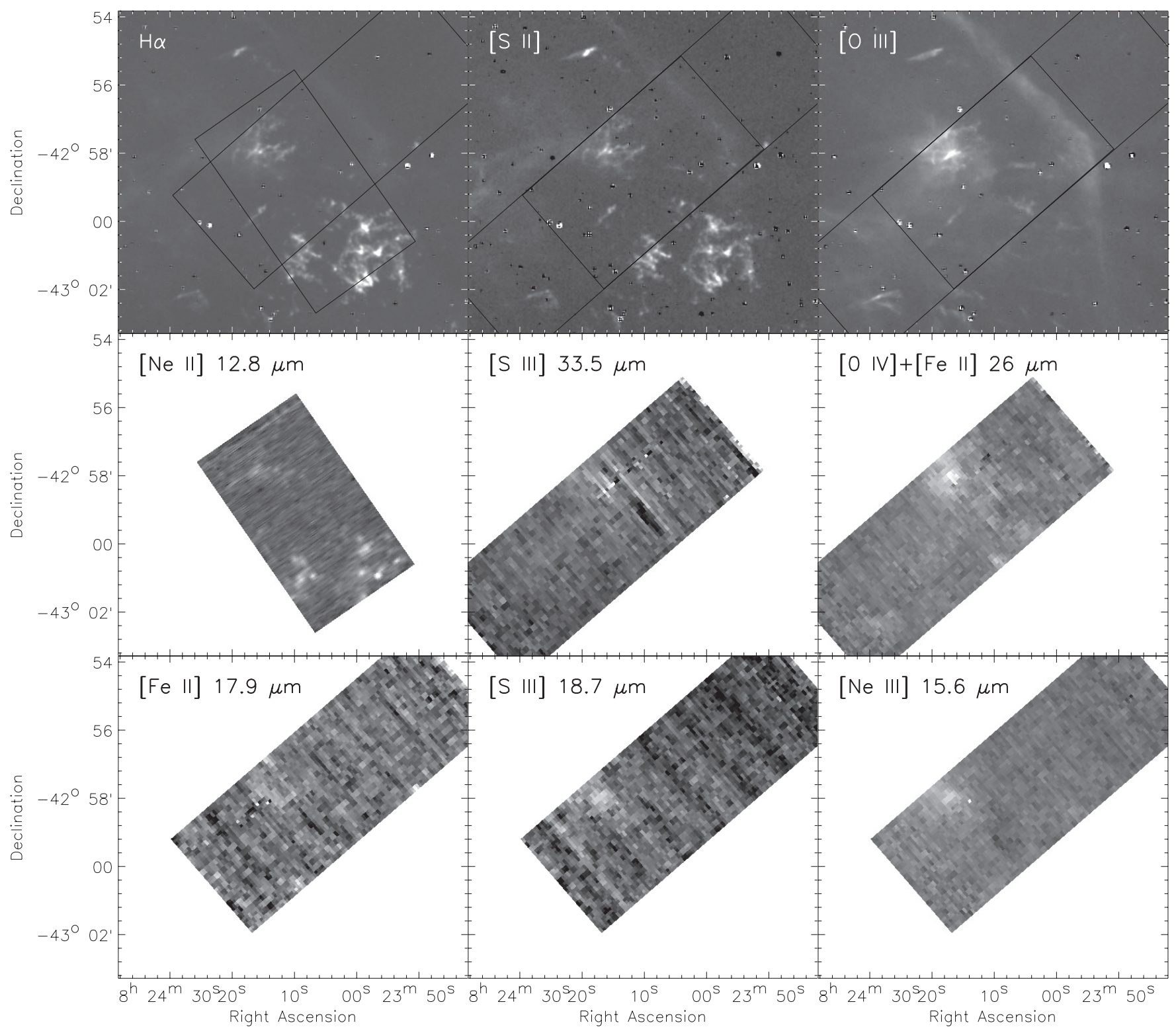

Figure 7. IR emission line images are compared to optical emission line images in the BEK region. IR lines from singly ionized species are well correlated with optical $\mathrm{H} \alpha$ and [S II]. Lines from doubly ionized species correlate well with optical [O III] emission. The $26 \mu \mathrm{m}$ line likely contains both [O IV] and [Fe II] because it correlates with all three of the optical lines. The [Ne II] spectral map is undersampled by a factor of 2 in the SE-NW dimension, and linear interpolation has been used to estimate the missing data.

(ISO) and falls below the sensitivity limits of the Midcourse Space Experiment (MSX) survey. The IRS spectra confirm that the broadband emission is dominated by thermal continuum emission of dust rather than atomic or molecular emission lines. The steep drop in the continuum at shorter wavelengths is a clear indication that small dust grains, which are directly confirmed to be present in the surrounding ISM, are absent within the shocked gas of the BEK. The comparison of the dust grains size distribution $[f(a)=d n / d a]$ in the ISM with the post-shock size distribution shows that $\sim 25 \%$ of the total dust mass has been destroyed in the BEK. This is depicted in Figure 10 where the pre-shock and post-shock distribution of $a^{4} f(a)(\propto a d m / d a$, with $d m / d a=$ the mass distribution of the dust grains) is plotted as a function of grain size, $a$, for each of the physical components (PAH, graphite, silicate) of the ZDA BARE-GR-FG model. The plot provides a good illustration of the fact that most of the mass of the dust resides in the largest dust grains despite the greater numbers of small grains. It also shows that under the action of sputtering, most of the mass loss occurs in the destruction of the smallest dust grains rather than the erosion of the largest grains. This is also shown in Figure 11 which demonstrates that the dust mass destroyed jumps quickly as sputtering begins and then increases more slowly as sputtering continues to larger values of $\Delta a$. For the Compact Knot region, Figure 8 shows that within the three-dimensional parameter space, models with $\chi^{2}<2 \min \left(\chi^{2}\right)$ have $0.0005<\Delta a<0.0050 \mu \mathrm{m}$. Thus, from Figure 11, we can see the corresponding mass fraction of graphite and silicate grains destroyed ranges from $15 \%$ to $35 \%$. The value of $\Delta a$, and thus the fractional mass destroyed, is less well constrained for the Bar- $\mathrm{N}$ and Bar-S regions.

Qualitatively similar results are obtained in a non-radiative shock examined by Sankrit et al. (2010) in the Cygnus Loop. There, with slightly lower post-shock electron densities and substantially lower temperatures, roughly $35 \%$ of the dust mass 

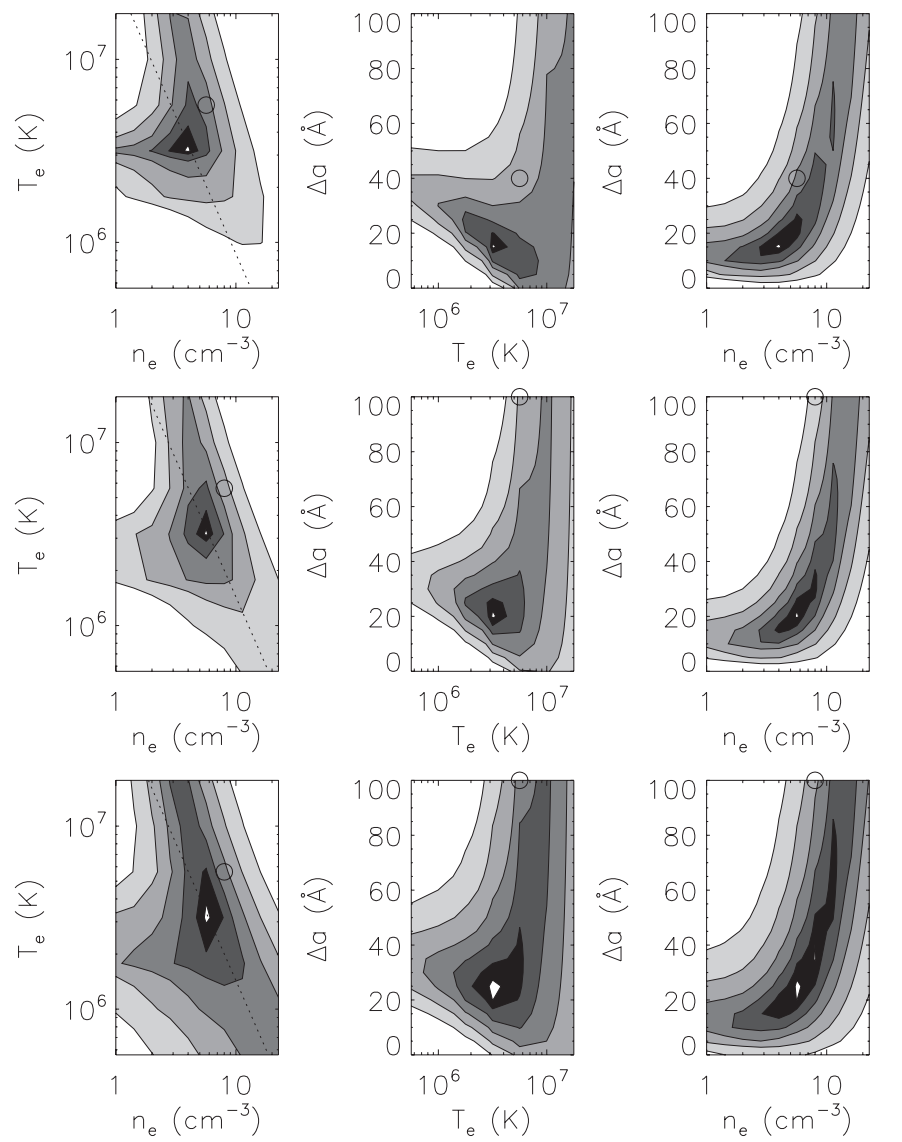

Figure 8. Slices of $\chi^{2}$ for fitting the Compact Knot, Bar-N, and Bar-S spectra (top to bottom) using the ZDA dust model. Each two-dimensional slice passes through the minimum value of $\chi^{2}$ in the three-dimensional $\left(T_{e}, n_{e}, \Delta a\right)$ parameter space. Contour levels $=[1.2,2,4,8,16,32] * \min \left(\chi^{2}\right)$. For the Compact Knot and Bar-N regions, the black circles identify the projection of the minimum $\chi^{2}$ onto these slices, when $T_{e}$ and $\Delta a$ are constrained by the results of the X-ray modeling of Hwang et al. (2005).

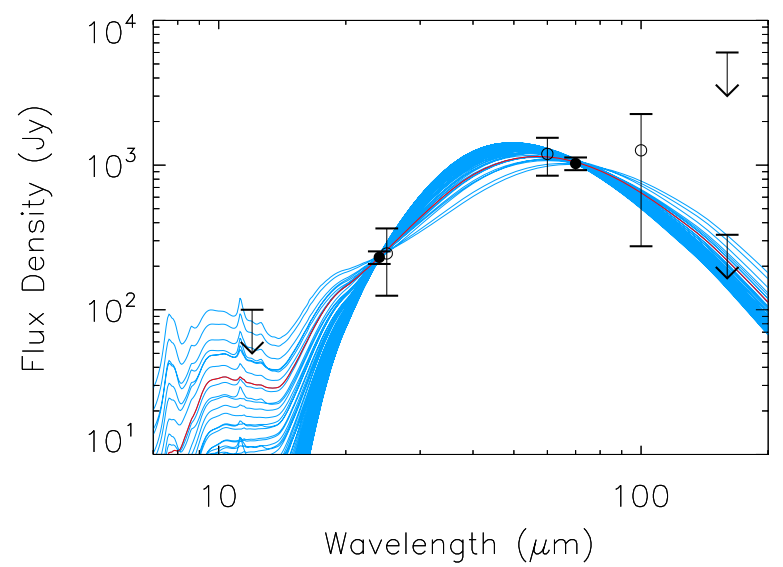

Figure 9. Integrated flux densities of the entire Puppis A SNR. Solid data points are Spitzer MIPS observations. Open data points are IRAS data from Arendt et al. (1991). The higher upper limit at 160 includes all emission across the SNR, most of it likely to be ISM. The fainter $160 \mu \mathrm{m}$ upper limit applies only to emission that is correlated with the 24 (and 70) $\mu \mathrm{m}$ emission. The red line indicates the best fitting ZDA dust model, while the blue lines indicate all the other models from the grid in $\left\{T_{e}, n_{e}, \Delta a\right\}$ which have a reduced $\chi^{2}<1$.

(A color version of this figure is available in the online journal.)

is destroyed over a spatial scale of $\sim 0.14 \mathrm{pc}$. This spatial scale is not well resolved in Puppis A, which is four times more distant than the Cygnus Loop.

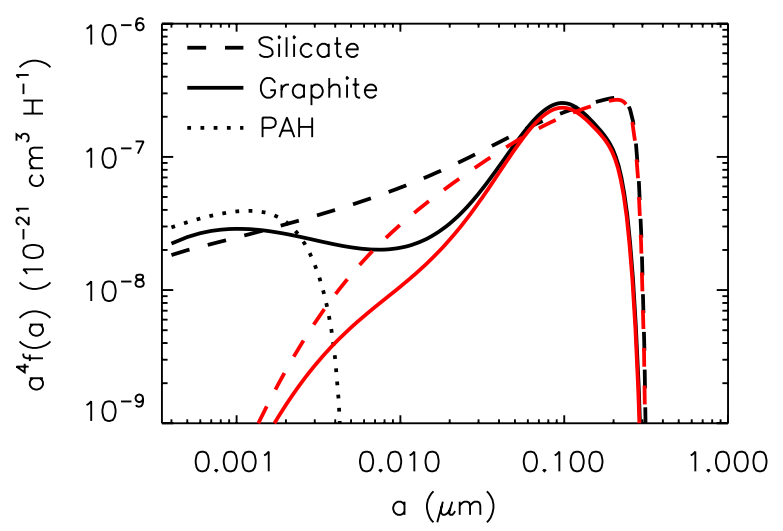

Figure 10. Mass distribution of ZDA dust grains before (black lines) and after sputtering by $\Delta a=0.002 \mu \mathrm{m}$ (red lines). The unsputtered mass distribution is that used to model the East Cloud. The sputtered mass distribution is appropriate for the Bar-N region. This amount of sputtering destroys $100 \%$ of the PAHs and removes $24 \%$ and $23 \%$ of the total mass in graphite and silicate grains, respectively.

(A color version of this figure is available in the online journal.)

In principle, the destruction of the dust may lead to an increase in the strength of emission lines associated with atoms that are highly depleted in the gas phase of the general ISM, e.g., Si and Fe. Emission of [Fe II] is detected at 17.9 and $26.0 \mu \mathrm{m}$; however, this emission is spatially correlated with optical filaments and knots visible in $\mathrm{H} \alpha$ and [S II] rather than the dust and X-ray emission. There are such structures near the Compact Knot, but none are present near the bright parts of the Bar. No emission from the [Si II] $34.8 \mu \mathrm{m}$ line is observed. This line is ubiquitous in the raw spectra, but then disappears completely when the background subtraction is applied to the data. The lack of IR emission lines from the sputtered atoms is likely due to the fact that the atoms are quickly ionized beyond the singly ionized stages which have strong lines in the range of the IRS spectral coverage. Itoh (1989) has modeled the time-dependent ionization of atoms sputtered from dust. His model 65D matches the $T_{e}=3 \times 10^{6} \mathrm{~K}$ environment of the BEK. This model indicates that most of the sputtered $\mathrm{Si}$ atoms proceed beyond single ionization after $n_{e} t>1 \mathrm{~cm}^{-3} \mathrm{yr}$. Thus, at the density of the BEK, freshly sputtered $\mathrm{Si}$ atoms can only produce the [Si II] $34.8 \mu \mathrm{m}$ line for $<0.25 \mathrm{yr}$.

The spectra of the Compact Knot and the Bar reveal no $\mathrm{PAH}$ emission, even after we implemented a fitting procedure that can recover oversubtraction of the background. Yet the IRAC imaging suggests weak 3.6-8 $\mu \mathrm{m}$ emission at the eastern (leading) edges of the bright $24 \mu \mathrm{m}$ and X-ray emission. The broadband colors of this emission are similar to those produced by PAHs, and the low surface brightness is consistent with the non-detection of PAHs in the spectra. The morphology and relative faintness compared to the $24 \mu \mathrm{m}$ emission suggest that the IRAC data are tracing the zone behind the shock front (or the precursor in front of the shock) in which the PAHs are being destroyed. This is consistent with the fact that PAHs should be completely destroyed in $v>120 \mathrm{~km} \mathrm{~s}^{-1}$ shocks (Micelotta et al. 2010).

As described in Section 2.1, the IRS observations of Puppis A only cover the region of the BEK, a swept-up interstellar cloud, and not any of the locations where knots or filaments of ejecta are found. Therefore, we cannot make any useful assessment of the amount of grain processing of dust formed in the SN ejecta. However, the detection of the Omega Filament in the $24 \mu \mathrm{m}$ MIPS data means that future spectroscopic observations 


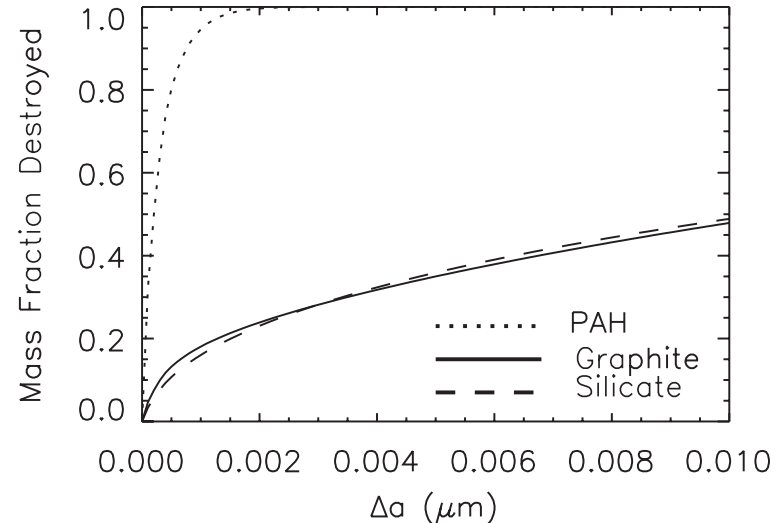

Figure 11. Fractional mass of ZDA dust grains (BARE-GR-FG model) eroded as a function of the total amount of sputtering $\Delta a$.

should be able to sort out the relative contributions of line and continuum emission, and provide characterization of the surviving $\mathrm{SN}$ ejecta dust.

\subsection{Characterization of the X-ray Emitting Gas}

In modeling the IR emission of collisionally heated dust, we have chosen to treat the gas properties as free parameters, rather than constraining them with results from modeling the X-ray spectra (e.g., Hwang et al. 2005). Thus, we obtain an independent characterization of the hot post-shock gas. The models of the X-ray spectra are characterized by the gas temperature $\left(T_{e}\right)$, an "ionization timescale" $\left(n_{e} t\right)$ which indicates the length of time since the gas was shocked via its approach to ionization equilibrium, and a line-of-sight column density $\left(N_{H}\right.$, not an intrinsic property of the SNR). The gas temperature is a direct parameter of the IR modeling, and the ionization timescale is proportional to $\Delta a$ via

$$
n_{e} t \approx n_{H} t \approx 6 \times 10^{12} \Delta a,
$$

where the proton and electron densities $n_{H}, n_{e}$, are in $\mathrm{cm}^{-3}$, $t$ is in $\mathrm{s}$, and $\Delta a$ is in $\mu \mathrm{m}$, for dust that is thermally and kinetically sputtered in hot gas $\left(T_{e}>10^{6} \mathrm{~K}\right)$ behind fast shocks $\left(v_{s}>500 \mathrm{~km} \mathrm{~s}^{-1}\right)$ (e.g., Dwek et al. 2008). In detail, the coefficient of this relation is dependent on the grain composition (i.e., sputtering yield), and whether or not kinetic sputtering is acting in addition to thermal sputtering.

The $3.2 \times 10^{6} \mathrm{~K}$ gas temperature that we derive in all three portions of the BEK is somewhat lower than the corresponding X-ray-derived values of $T_{e} \approx 5-7.4 \times 10^{6} \mathrm{~K}$ across the Compact Knot and Bar regions (see Table 2). The inferred values of $\Delta a$ are significantly lower than the values that are indicated by the ionization timescale, $n_{e} t$, of the $\mathrm{X}$-ray modeling. Using Equation (1) with the IR-derived sputtering and gas density allows us to estimate the time since the dust was swept up as $t \approx 70-90 \mathrm{yr}$. This age is far shorter than the $\sim 2000 \mathrm{yr}$ age derived by Hwang et al. (2005) based on comparison of the X-ray spectrum and morphology to shocked clouds models calculated by Klein et al. (2003). The IR sputtering timescale is also shorter than the 600-1200 yr timescale derived by dividing the X-ray-derived ionization timescale by the IR-derived gas density. Here, we assume that the mean $n_{e} t$ is half of the maximum $n_{e} t$ derived from the X-ray spectral models, which are for plane-parallel single-temperature shocks and hence integrate the emission over a range of $n_{e} t$.
The remaining discrepancy suggests that the dust sputtering rate of Equation (1) is overestimated, either because kinetic sputtering plays a smaller than expected role or because the sputtering yields are smaller. Additionally, the ages derived from dust sputtering may differ from the X-ray-derived ages because the interaction with the East Cloud is an ongoing process, not an instantaneous event that occurred at a specific date. In detail, the models should be integrated over time, weighted by the rate at which mass was swept up $(d M / d t)$. Over time, the dust is sputtered and the gas cools. The sputtering of the dust occurs on shorter timescales, and thus the integral of the IR emission over time will be more heavily weighted toward the most recently swept-up ISM where the small grains are still present.

Consistency between the IR and X-ray analyses can be strengthened by adopting the X-ray-derived $T_{e}$ and $n_{e} t$ (and thus $\Delta a$ ) as fixed parameters of the dust models. The work by Hwang et al. (2005) includes a detailed examination of a slice across the BEK passing through the Compact Knot and the Bar-N regions. Their analysis reveals that $T_{e}$ rises by a factor of 2 from the east to west side of the Compact Knot and then remains relatively constant across the Bar. The ionization timescale $n_{e} t$ is low $\left(\sim 0.5 \times 10^{11} \mathrm{~s} \mathrm{~cm}^{-3}\right)$ across the east side and center of the Compact Knot, with a sharp jump to higher values $\left(\sim 2.5 \times 10^{11} \mathrm{~s} \mathrm{~cm}^{-3}\right)$ occurring on the western edge of the Compact Knot. This jump occurs within the region averaged for the IR spectrum of the Compact Knot. The ionization timescale remains high across the Bar. We have examined these models to determine the mean $T_{e}$ and $n_{e} t$ over the three regions corresponding to our IR spectra (Table 2 ). Based on these results, if we restrict our grid of IR models to $T_{e}=5.6 \times 10^{6} \mathrm{~K}$ and $\Delta a=0.0040-0.0100 \mu \mathrm{m}$ (the range allows for the jump in $n_{e} t$ ) for the Compact Knot and $T_{e}=5.6 \times 10^{6} \mathrm{~K}$ and $\Delta a=0.0100 \mu \mathrm{m}$ for the Bar regions, then the remaining free parameter of the models, $n_{e}$, is found to increase relative to the best-fitting models to values of $n_{e}=5.7$ and 8 for the Compact Knot and Bar regions, respectively. However, the quality of the fits suffers. The increased amount of sputtering further reduces the contribution of small dust grains, which are responsible for the short wavelength emission. To compensate for the weaker short wavelength emission, the derived gas density is higher, which raises the temperature of the remaining larger grains. Yet the resulting spectra still fall too steeply at short wavelengths $(\lambda<20 \mu \mathrm{m})$, and the spectra fall too steeply at the longer wavelengths $(25<\lambda<70 \mu \mathrm{m})$ as well. When thus constrained, the model spectra become too sharply peaked because of the lack of the smaller grains with their widely fluctuating temperatures.

\subsection{Other Sources}

X-ray observations have revealed a neutron star, RXJ08224300, associated with the Puppis A SNR (e.g., Petre et al. 1996; Winkler \& Petre 2007; Gotthelf \& Halpern 2009). However, this object has not been detected at optical wavelengths or in deep 4.5 and $8 \mu \mathrm{m}$ observations with IRAC (Wang et al. 2007; Mignani et al. 2009). Likewise, in our shallow MIPS observations, there is no detection of any associated emission at 24,70 , or $160 \mu \mathrm{m}$. At $24 \mu \mathrm{m}$, the $1 \sigma$ variation of the local background is $\sim 0.25 \mathrm{MJy} \mathrm{sr}^{-1}$, which implies a $1 \sigma$ point source sensitivity of $\sim 0.24 \mathrm{mJy}$.

The IRAC and MIPS images incidentally reveal several very distinctive objects which are unassociated with Puppis A, e.g., (1) a symmetric 16" radius edge-on galaxy at $(\alpha, \delta)=\left(8^{\mathrm{h}} 24^{\mathrm{m}} 13^{\mathrm{s}},-42^{\circ} 56^{\prime} 18^{\prime \prime}\right)$ is clearly seen at all four IRAC wavelengths. This galaxy is faintly visible in Two Micron 
All Sky Survey images at $K, H$, and (barely) $J$ bands. At $24 \mu \mathrm{m}$, any emission from the galaxy is lost behind the brighter emission of Puppis A. (2) The MIPS $24 \mu \mathrm{m}$ image contains several compact clusters of point sources in the northern half of the field. The brightest objects in the clusters are usually detected at $70 \mu \mathrm{m}$ as well. The most prominent of these clusters contains more than ten $24 \mu \mathrm{m}$ sources within a $2^{\prime}$ radius, including IRAS 08222-4214 and IRAS 08222-4212. Another cluster (identified by Dutra et al. 2003) and its associated reflection nebula produce the extended and extremely bright source at $(\alpha, \delta)=\left(8^{\mathrm{h}} 26^{\mathrm{m}} 26^{\mathrm{s}},-42^{\circ} 32^{\prime} 40^{\prime \prime}\right)$. (3) The bright linear $160 \mu \mathrm{m}$ cloud, about $15^{\prime}$ long at $(\alpha, \delta)=\left(8^{\mathrm{h}} 22^{\mathrm{m}} \cdot 2,-43^{\circ} 21^{\prime \prime}\right)$, is a dark cloud at visible wavelengths. The facts that it is a visible dark cloud (Feitzinger \& Stüwe 1984) and that the measured velocity of its CO emission is $+6 \mathrm{~km} \mathrm{~s}^{-1}$ (Ramesh 1994), compared to $\sim+16 \mathrm{~km} \mathrm{~s}^{-1}$ for clouds associated with the SNR, both indicate that this cloud lies well in the foreground of Puppis A.

\section{SUMMARY}

Spitzer MIPS images at 24 and $70 \mu \mathrm{m}$ show that the IR to X-ray correlation within the Puppis A SNR is very strong on all spatial scales. The total IR luminosity of the SNR is $\sim 1.4 \times 10^{4} L_{\odot}$ produced by $0.25 M_{\odot}$ of dust. The ratio of IR to X-ray emission is 6.9 , indicating that the IR emission is the dominant radiative energy loss of the SNR. At $160 \mu \mathrm{m}$ the SNR becomes lost in the confusion of the Galactic plane indicating that there is not a large column density of colder dust associated with the SNR. At the BEK, IRAC images show weak emission that has colors similar to the PAH emission of the general ISM. This may indicate the zone where PAHs are destroyed in the shock front. Some 5.8 and $8 \mu \mathrm{m}$ emission associated with optical emission of singly ionized species is also noted in a region south of the BEK. Across the SNR, there are only a small number of locations where optical filaments have IR counterparts in the broadband MIPS images. The most prominent of these is the oxygen rich Omega filament.

The IRS, SL, and LL spectra of the East Cloud outside the SNR reveal a typical ISM spectrum, dominated by PAH emission features. The longer wavelength thermal emission of the larger graphite and silicate grains suggests an ISRF that is $\sim 10$ times stronger than the local ISRF in the solar vicinity. The spectral map of the BEK region does not show PAH features, but does show the characteristic silicate band at $20 \mu \mathrm{m}$. The external grain size distribution must be subjected to sputtering to reduce grain sizes by $\Delta a \approx 0.002 \mu \mathrm{m}$ in order to fit the observed BEK spectrum. This implies an exposure timescale of $\sim 80 \mathrm{yr}$, which is shorter than timescales estimated from prior analysis of the X-ray spectra and morphology, indicating a bias toward emission from the more recently swept-up dust in a process of extended duration. In the region of the BEK, the $500 \mathrm{~km} \mathrm{~s}^{-1}$ shocks have destroyed $\sim 25 \%$ of the mass of the swept-up interstellar dust. This fraction may be as low as $15 \%$ or as high as $35 \%$, as it is derived from the fitted value of $\Delta a$ which is strongly correlated with the temperature and density of the gas that collisionally heats and sputters the dust grains. The amount of dust destroyed will increase until the post-shock gas cools and sputtering becomes ineffective.

This work is based on observations made with the Spitzer Space Telescope, which is operated by the Jet Propulsion Laboratory, California Institute of Technology under a contract with NASA. Support for this work was provided by
NASA. We acknowledge the use of NASA's SkyView facility (http://skyview.gsfc.nasa.gov) located at NASA Goddard Space Flight Center. This research has made use of NASA's Astrophysics Data System Bibliographic Services. P.F.W. acknowledges additional funding from the NSF through grant AST0908566.

\section{REFERENCES}

Arendt, R. G. 1989, ApJS, 70, 181

Arendt, R. G., Dwek, E., \& Petre, R. 1991, ApJ, 368, 474

Arendt, R. G., Dwek, E., Petre, R., Dickel, J. R., Roger, R. S., Milne, D. K., \& Kesteven, M. J. 1990, ApJ, 350, 266

Arendt, R. G., et al. 2008, ApJ, 682, 384

Baade, W., \& Minkowski, R. 1954, ApJ, 119, 206

Castelletti, G., Dubner, G., Golap, K., \& Goss, W. M. 2006, A\&A, 459, 535

Cherchneff, I., \& Dwek, E. 2010, ApJ, 713, 1

Dubner, G. M., \& Arnal, E. M. 1988, A\&AS, 75, 363

Dutra, C. M., Bica, E., Soares, J., \& Barbuy, B. 2003, A\&A, 400, 533

Dwek, E. 1998, ApJ, 501, 643

Dwek, E., Galliano, F., \& Jones, A. P. 2007, ApJ, 662, 927

Dwek, E., Petre, R., Szymkowiak, A., \& Rice, W. L. 1987, ApJ, 320, L27

Dwek, E., et al. 2008, ApJ, 676, 1029

Fazio, G. G., et al. 2004, ApJS, 154, 10

Feitzinger, J. V., \& Stüwe, J. A. 1984, A\&AS, 8, 365

Fixsen, D. J., Moseley, S. H., \& Arendt, R. G. 2000, ApJS, 128, 651

Gehrz, R. D., et al. 2007, Rev. Sci. Instrum., 78, 011302

Gotthelf, E. V., \& Halpern, J. P. 2009, ApJ, 695, L35

Goudis, G., \& Meaburn, J. 1978, A\&A, 62, 283

Houck, J. R., et al. 2004, ApJS, 154, 18

Hwang, U., Flanagan, K. A., \& Petre, R. 2005, ApJ, 635, 355

Hwang, U., Petre, R., \& Flanagan, K. A. 2008, ApJ, 676, 378

Itoh, H. 1989, PASJ, 41, 853

Jones, A. P. 2004, in ASP Conf. Ser. 309, Astrophysics of Dust, ed. A. N. Witt, G. C. Clayton, \& B. T. Draine (San Francisco, CA: ASP), 347

Jones, A. P., Tielens, A. G. G. M., \& Hollenbach, D. J. 1996, ApJ, 469, 740

Katsuda, S., Hwang, U., Petre, R., Park, S., Mori, K., \& Tsunemi, H. 2010, ApJ, 714, 1725

Katsuda, S., Mori, K., Tsunemi, H., Park, S., Hwang, U., Burrows, D. N., Hughes, J. P., \& Slane, P. O. 2008, ApJ, 678, 297

Klein, R. I., Budil, K. S., Perry, T. S., \& Bach, D. R. 2003, ApJ, 583, 245

Maiolino, R., Schneider, R., Oliva, E., Bianchi, S., Ferrara, A., Mannucci, F., Pedani, M., \& Roca Sogorb, M. 2004, Nature, 431, 533

Makovoz, D., \& Marleau, F. R. 2005, PASP, 117, 1113

Mathis, J. S. 1990, ARA\&A, 28, 37

Mathis, J. S., Mezger, P. G., \& Panagia, N. 1983, A\&A, 128, 212

Micelotta, E. R., Jones, A. P., \& Tielens, A. G. G. M. 2010, A\&A, 510, A36

Mignani, R. P., de Luca, A., Mereghetti, S., \& Caraveo, P. A. 2009, A\&A, 500, 1211

Morgan, H. L., \& Edmunds, M. G. 2003, MNRAS, 343, 427

Morse, J. A., et al. 1996, AJ, 112, 509

Neugebauer, G., et al. 1984, ApJ, 278, L1

Paron, S., Dubner, G., Reynoso, E., \& Rubio, M. 2008, A\&A, 480, 439

Petre, R., Becker, C. M., \& Winkler, P. F. 1996, ApJ, 465, L43

Ramesh, B. 1994, JA\&A, 15, 415

Reach, W. T., et al. 2006, AJ, 131, 1479

Reynoso, E. M., Dubner, G. M., Goss, W. M., \& Arnal, E. M. 1995, AJ, 110, 318

Rieke, G. H., et al. 2004, ApJS, 154, 25

Saken, J. M., Fesen, R. A., \& Shull, J. M. 1992, ApJS, 81, 715

Sankrit, R., et al. 2010, ApJ, 712, 1092

Smith, J. D. T., et al. 2007, PASP, 119, 1133

Sofia, U. J., \& Meyer, D. M. 2001, ApJ, 554, L221

Sutherland, R. S., \& Dopita, M. A. 1995, ApJ, 439, 365

Tielens, A. G. G. M. 1998, ApJ, 499, 267

Valiante, R., Schneider, R., Bianchi, S., \& Andersen, A. C. 2009, MNRAS, 397, 1661

Wang, Z., Kaplan, D. L., \& Chakrabarty, D. 2007, ApJ, 655, 261

Werner, M. W., et al. 2004, ApJS, 154, 1

Winkler, P. F., \& Kirshner, R. P. 1985, ApJ, 299, 981

Winkler, P. F., \& Petre, R. 2007, ApJ, 670, 635

Winkler, P. F., Tuttle, J. H., Kirshner, R. P., \& Irwin, M. J. 1988, in IAU Colloq. 101, Supernova Remnants and the Interstellar Medium, ed. R. S. Roger \&

T. L. Landecker (Cambridge: Cambridge Univ. Press), 65

Zubko, V., Dwek, E., \& Arendt, R. G. 2004, ApJS, 152, 211 (ZDA) 